\title{
Actitudes docentes y buenas prácticas con TIC del profesorado de Educación Permanente de Adultos en Andalucía
}

\section{Teacher attitudes and best practices with ICT faculty Adult Continuing Education in Andalusia}

\author{
Jose María FERNÁNDEZ BATANERO y José Antonio TORRES GONZÁLEZ \\ Universidad de Sevilla y Universidad de Jaén
}

Recibido: Diciembre 2013

Aceptado: Septiembre 2014

\begin{abstract}
Resumen
El presente artículo recoge los resultados de un estudio cuyo objetivo ha sido determinar las actitudes del profesorado de educación permanente de adultos de la comunidad autónoma de Andalucía, hacia el uso e integración de las Tecnologías de la Información y Comunicación (TIC) en sus respectivos centros, al mismo tiempo que identificar aquellos factores que favorecen el desarrollo de buenas prácticas. El diseño de investigación es de tipo descriptivo mixto. Las técnicas utilizadas para la recogida de información han sido el cuestionario y la entrevista en profundidad. Se realizaron 172 encuestas a profesores y profesoras, así como 18 entrevistas a directores y coordinadores de Sección de Adultos. Para la validación del cuestionario, se utilizó la técnica de juicio de expertos, seleccionados mediante el procedimiento de «Coeficiente de competencia experta» o «Coeficiente K». Para fortalecer las propiedades psicométricas se determinó la validez de constructo mediante un análisis factorial con el método Varimax y extracción de máxima verosimilitud, donde se extrajeron dos factores. La confiablidad se estableció mediante el Alfa de Cronbach (0.88). La guía de entrevista fue validada por el mismo grupo de expertos. Los resultados señalan por un lado, que los docentes poseen actitudes positivas hacia las TIC tanto en lo relativo a su papel en desarrollo profesional, como en cuanto a la facilidad y accesibilidad de estas. Por otro, entre los factores más importantes para proporcionar buenas prácticas educativas con apoyo de TIC se encuentra la capacidad de estas para favorecer el trabajo personalizado.
\end{abstract}

Palabra clave: actitudes, adultos, tecnología, educación de adultos, educación tecnológica, buenas prácticas, competencias, educación. 


\begin{abstract}
The present paper reports the results of a study aiming to describe the attitudes of teachers in adult continuous education in the Autonomous Community of Andalusia (Spain) towards the use and integration of information and communication technologies (ITC) in the educational centres they work in, while identifying those factors that favour the development of good practice. It is a mixed methods descriptive research, and information collection techniques include a questionnaire and in-depth interviews. A total number of 172 teachers were surveyed, as well as 18 head teachers and coordinators, in adult education. For questionnaire validation the expert judgment technique was used, as they were selected by the «expert competence coefficient» or «K coefficient» procedure. To improve its psychometric properties, construct validity was determined by means of Varimax factor analysis and maximum likelihood extraction (two factors were extracted). Confidence was set by Cronbach's alpha (0.88). The interview guide was also validated by this group of experts. Results point out, on one hand, that teachers hold positive attitudes towards ICT regarding both ICT's role in professional development and their ease of use and access. On the other hand, among the most important factors for ICT-supported good educational practices lies in ICT's capacity to favour personalized work.
\end{abstract}

Keywords: attitudes, adults, technology, adult education, technology education, best practices, skills, education.

En este artículo se presentan los hallazgos obtenidos en un estudio que ha tenido por objetivo principal describir las actitudes de docentes hacia las TIC, que desarrollan su labor profesional en centros de educación permanente de adultos, al mismo tiempo que identificar aquellos factores que favorecen el desarrollo de buenas prácticas.

El texto está conformado por seis apartados: el primero describe algunas consideraciones sobre docentes, TIC y buenas prácticas en los procesos de enseñanza aprendizaje, así como el establecimiento del problema y la justificación del estudio. En el segundo se presenta el abordaje metodológico basado en un diseño de investigación descriptivo de tipo mixto, con una combinación de enfoques cuantitativo y cualitativo que permite identificar y explicar dichas actitudes, y el desarrollo de buenas prácticas con TIC. En el tercero se exponen los resultados obtenidos tanto del cuestionario como de las entrevistas en profundidad, estableciéndose las relaciones entre las puntuaciones obtenidas por el profesorado y los diferentes factores objeto de estudio en el cuestionario, así como el análisis de contenido relativo a las entrevistas. El cuarto se dedica a las conclusiones y su discusión con respecto a otros estudios similares. Finalmente en el quinto y sexto apartado se exponen las limitaciones del estudio y las posibles recomendaciones para futuras investigaciones.

\title{
Establecimiento del problema y justificación del estudio
}

Actualmente, la educación permanente de personas adultas tiene la finalidad de ofrecer a todas las personas mayores de dieciocho años la posibilidad de adquirir, actualizar, completar o ampliar sus conocimientos y aptitudes para el desarrollo personal o profesional. Desde el ámbito competencial la formación de personas adultas 
abarca todas las etapas educativas, excepto la universitaria, y los planes educativos que imparten incluyen planes de formación básica y planes educativos de enseñanzas no formales.

Es en este contexto educativo, donde las políticas de aprendizaje permanente se asocian a mercados de trabajo cambiantes, en los que hay que mantener viva la cualificación, adquirir nuevas competencias y ser capaz de cambiar a nuevas profesiones, y se vinculan a profundas transformaciones sociales, que exigen competencias personales para ser ciudadanos saludables, creativos, interactivos y comprometidos. El aprendizaje de personas adultas se convierte en estas circunstancias en una manera de lograr la mejora de la cualificación de las personas poco cualificadas o cuyas aptitudes profesionales se han quedado desfasadas, permitiéndoles adquirir competencias clave en cualquier momento de su vida.

Dicho aprendizaje convive con una serie de avances tecnológicos que hacen que los procesos de comunicación estén mediados por las Tecnologías de la Información y la Comunicación y que van a condicionar de manera directa el uso sistemático de estas tecnologías y el desarrollo futuro de la formación de personas adultas, tal como se desprende del Programa Internacional para la Evaluación de las Competencias de la Población Adulta (PIAAC, 2013). Ante esta situación, se precisa una formación para personas adultas que facilite el acceso a los recursos que la sociedad le presenta y no se quede en lo meramente instrumental-académico.

Consideramos que este estudio es importante por varias razones, en primer lugar, a pesar de que la línea de investigación acerca de las actitudes docentes viene desarrollándose desde hace más de dos décadas, todavía hoy en día, son muy escasos en los estudios existentes que verifiquen las actitudes del profesorado de educación de adultos hacia las TIC en el marco educativo. Una segunda razón obedece a aspectos sociales, donde los procesos de discriminación y exclusión social en la población adulta son de especial preocupación en familias, centros educativos, ayuntamientos y otras instituciones con incidencia en la formación ciudadana; por último, reparar en los factores de eficiencia y eficacia de las prácticas pedagógicas con TIC implica que se está pensando en consolidar procesos de innovación y calidad educativa, siempre con el propósito de hacer de lo excepcional, que es el uso de las tecnologías, algo cotidiano, fortaleciendo los aprendizajes y las relaciones interpersonales entre los agentes educativos del centro.

En definitiva, este trabajo resulta interesante, además, porque permite analizar no sólo las actitudes docentes hacia estas tecnologías en aulas de educación de personas adultas, sino que también se estará en condiciones de buscar evidencias que permitan argumentar la existencia de factores favorecedores del aprendizaje con TIC, que potencian el desarrollo de buenas prácticas educativas.

Partiendo de este contexto nos hemos planteado las siguientes preguntas de investigación:

- ¿Qué actitudes presentan los docentes andaluces de educación permanente de adultos hacia las TIC? 
- ¿Qué factores favorecen el desarrollo de buenas prácticas educativas de enseñanza-aprendizaje con apoyo TIC?

\section{Antecedentes y fundamentación teórica}

Hoy día, los entornos de aprendizaje tradicionales centrados en el docente se manifiestan como insuficientes para favorecer las finalidades educativas de la mayoría de los sistemas educativos contemporáneos apoyados con TIC. En esta línea, satisfacer las necesidades educativas de la sociedad actual implica cambiar la imagen de una enseñanza fundamentalmente centrada en el profesorado, para dibujar entornos de enseñanza diversificados en que se comience a considerar con seriedad el papel del alumnado, el nuevo modo de construir el conocimiento, la posibilidad real de llevar a cabo evaluaciones más eficaces y el contacto más directo e interactivo con la comunidad. Así pues, una de las conclusiones más destacables de los estudios actuales es que, a pesar del incremento de la disponibilidad de recursos tecnológicos en los centros educativos, la práctica pedagógica de los docentes en el aula no supone necesariamente una modificación sustantiva del modelo de enseñanza tradicional (Área, 2008; 2010). Aunque las TIC pueden constituir un eficaz instrumento para mejorar la educación, es necesario que lleve aparejada una transformación de las actuaciones docentes, ya que el aprendizaje de los alumnos se relaciona con la calidad de las prácticas en las que participan dentro del aula (Coll, Mauri \& Onrubia, 2008).

De todos los factores que pueden incidir en dicha transformación, existen dos que pueden ejercer un mayor influjo. Por un lado, el grado de formación tecnológica del profesorado, a pesar de que en la actualidad aún son muchos los profesores que no se consideran competentes para abordar la integración de las TIC en sus prácticas docentes y que, en consecuencia, no han descubierto la relevancia de estos nuevos medios para el aprendizaje. Por consiguiente, podemos deducir que programas fallan en la preparación adecuada del profesorado, ya que no establecen conexiones pedagógicas entre las posibilidades de las tecnologías y la enseñanza de un particular contenido curricular (Valverde, Garrido \& Fernández Sánchez, 2010).

Por otro lado, la actitud del profesorado, pudiendo ser esta también una de las razones por las que las tecnologías no se utilicen en el aula, lo que conlleva a una falta de aprovechamiento de los beneficios que éstas tecnologías pueden aportar, tanto a su trabajo como al aprendizaje de sus alumnos (Hinojo \& Fernández Granada, 2002). En esta línea, la mayoría de estudios en donde se trata de obtener el grado de implantación de las TIC en los centros educativos incluyen un apartado referido a las actitudes del profesorado (Cabero, 1991; Castaño, 1994; Rodríguez Mondejar, 2000; Van Braak, 2001; Barajas, Scheuermann, \& And Kikis, 2002; Orellana, Almerich, Belloch \& Díaz, 2009; Galanouli, Murphy \& Gardner, 2004; Mooij, 2004; Gargallo, Suárez, Belloch, et al., 2004; Tejedor, García-Valcarcel \& Prada, 2009; Sáez López, 2010; Boza, Tirado \& Guzmán, 2010; Fernández Batanero \& Bermejo, 2012; Valdés, Angulo, Nieblas, Zambrano \& Arreola, 2012) ya que son un buen predictor de la implantación, integración y uso de las TIC en la educación. 
Para hacer frente a lo anterior, el profesorado precisa de nuevas competencias y modelos de trabajo no requeridos con anterioridad. En esta vertiente formativa el conocimiento y divulgación de "buenas prácticas" constituye una de las opciones de interés que permiten apoyar la integración real de las TIC en los procesos de enseñanza. En el contexto andaluz se contempla el concepto de buenas prácticas como "aquellas que explotan las nuevas herramientas tecnológicas para conseguir en el alumnado un aprendizaje autónomo que le motive a estar en permanente aprendizaje a lo largo de toda su vida" (Palomo, Ruiz y Sánchez, 2006: 24). El termino de "buenas prácticas" con apoyo de TIC no es un concepto nuevo, ya autores como Chickering y Gamson (1987), en un trabajo ya clásico, formularon una propuesta en la que se identifican hasta siete principios que configuran una buena práctica educativa: promueve las relaciones entre profesores y alumnos; desarrolla dinámicas de cooperación entre los alumnos; aplica técnicas activas para el aprendizaje; permite procesos de retroalimentación; enfatiza el tiempo de dedicación a la tarea; comunica altas expectativa, y por último, respeta la diversidad de formas de aprender.

En este sentido, la identificación de buenas prácticas permite aprender de las mismas, contextualizarlas y mejorar las propias prácticas, haciendo posible su transferencia a otros contextos. Así, más recientemente Epper y Bates (2004) citan las siguientes características para referirse a las buenas prácticas: contribuyen a mejorar el desempeño de un proceso; responden a una experiencia sistematizada, documentada y experimentada; su diseño se realiza desde un enfoque innovador; que aplican métodos de excelencia basados en la innovación; la categoría de buenas prácticas las hace extrapolables a otros contextos.

Para concluir, podemos decir que la identificación y selección de buenas prácticas, así como su difusión a la comunidad educativa, permitirán optimizar o mejorar los resultados futuros en las aulas de formación permanente de adultos.

\section{Diseño y metodología}

Teniendo en cuenta tales interrogantes, el proyecto de investigación se propuso como objetivo del estudio describir las actitudes de los docentes, que desarrollan su labor profesional en centros de educación permanente de adultos, hacia las TIC, e identificar aquellos factores que favorecen el desarrollo de buenas prácticas educativas de enseñanza-aprendizaje con apoyo TIC. Más concretamente la exploración se concretó en los siguientes objetivos específicos:

- Describir las actitudes que tienen los docentes hacia el uso de las TIC en los aspectos relativos a la Facilidad y disponibilidad, así como Apoyo en la práctica docente y formación.

- Establecer si hay relaciones significativas entre las actitudes de los docentes hacia las TIC y las variables sociodemográficas (edad y género) y posibilidades de interacción con ellas.

- Establecer si hay relaciones significativas entre las actitudes de los docentes hacia las TIC en función del programa que imparten. 
- Identificar posibles factores que propician el desarrollo de buenas prácticas educativas de enseñanza y aprendizaje con apoyo TIC.

Para dar respuesta a los problemas de investigación planteados, optamos por un diseño de investigación descriptivo de tipo mixto (metodología cuantitativa y cualitativa). Las técnicas destinadas a la recogida de la información han sido el cuestionario y las entrevistas en profundidad.

\section{Población y muestra}

La población de profesores y profesoras, coordinadores y directores de Centros y Secciones de Educación Permanente (CEPER), en septiembre de 2012 ${ }^{1}$, que imparten enseñanzas en planes educativos de Formación Básica, niveles I y II, en la provincia de Cádiz, Córdoba, Huelva y Sevilla era de 674, repartidos en 53 centros y 76 secciones de educación permanente. Del total de profesores y profesoras a los que se les envió el cuestionario, respondieron 172 , lo que representa el $25,51 \%$ que, para un nivel de confianza del 95,5\%, supone un error de muestreo inferior al 3\%. Este error es relativamente bajo ya que generalmente se asume un error del $5 \%$.

Con respecto a las entrevistas, se realizaron 18 entrevistas en profundidad (5 directores y 13 coordinadores de secciones). Para el tratamiento y análisis de la información se optó por la categorización, utilizando para ello una herramienta informática que facilita el análisis cualitativo de datos, el Nudist Vivo 7.0.

\begin{tabular}{lcccc} 
& Cádiz & Córdoba & Huelva & Sevilla \\
\hline Cuestionarios & 43 & 31 & 24 & 74 \\
\hline Entrevistas & 3 & 4 & 5 & 6 \\
\hline
\end{tabular}

Tabla 1. Cuestionarios y entrevistas por provincias

\section{Instrumentos}

El cuestionario dirigido al profesorado es una adaptación del utilizado por Fernández-Batanero \& Bermejo (2012) para docentes de educación secundaria en centros de orientación inclusiva. Dicho instrumento se contestó en una escala tipo Likert con cinco opciones que van desde Totalmente de Acuerdo (5), De acuerdo, No puedo opinar, Desacuerdo y Totalmente en Desacuerdo (1). El cuestionario consta de tres dimensiones: datos de identificación; apoyo en el desarrollo profesional y docente; $\mathrm{y}$, facilidad y disponibilidad. Para la validación del cuestionario (validez de contenido), se seleccionó a un grupo de expertos compuesto por 10 profesores universitarios, de los que 6 eran especialistas en Tecnología Educativa y 4 en Educación de Personas Adultas. Para su selección se utilizó el procedimiento de «Coeficiente de competencia experta» $\mathrm{o}$ «Coeficiente $\mathrm{K}$ » obtenido mediante la aplicación de la siguiente fórmula: $\mathrm{K}$ $=1 / 2(\mathrm{Kc}+\mathrm{Ka})$, donde $\mathrm{Kc}$ es el «Coeficiente de conocimiento» o información que tiene el experto acerca del tema o problema planteado; y $\mathrm{Ka}$ es el denominado «Coeficiente de argumentación» o fundamentación de los criterios de los expertos

\footnotetext{
${ }^{1}$ Datos proporcionados por el Servicio de Educación Permanente de la Consejería de Educación de la Junta de Andalucía.
} 
(Blasco et al., 2010). En nuestro caso, el coeficiente $\mathrm{K}$ fue superior a 0,8 en ocho de los diez expertos seleccionados, denotando ello un grado de competencia muy aceptable. Las estimaciones de los expertos se realizaron en sucesivas rondas, anónimas, al objeto de tratar de conseguir consenso, pero con la máxima autonomía por parte de los participantes (método Delphi).

Para determinar la validez del constructo, se realizó un análisis factorial con el método de rotación ortogonal. Se alcanzó un KMO de .86 , lo cual habla de la adecuación del modelo y una prueba de esfericidad de Bartlett significativa $(\mathrm{p}=.000)$. Se extrajeron dos factores que explican el $48,8 \%$ de la varianza del constructo (Tabla 2).

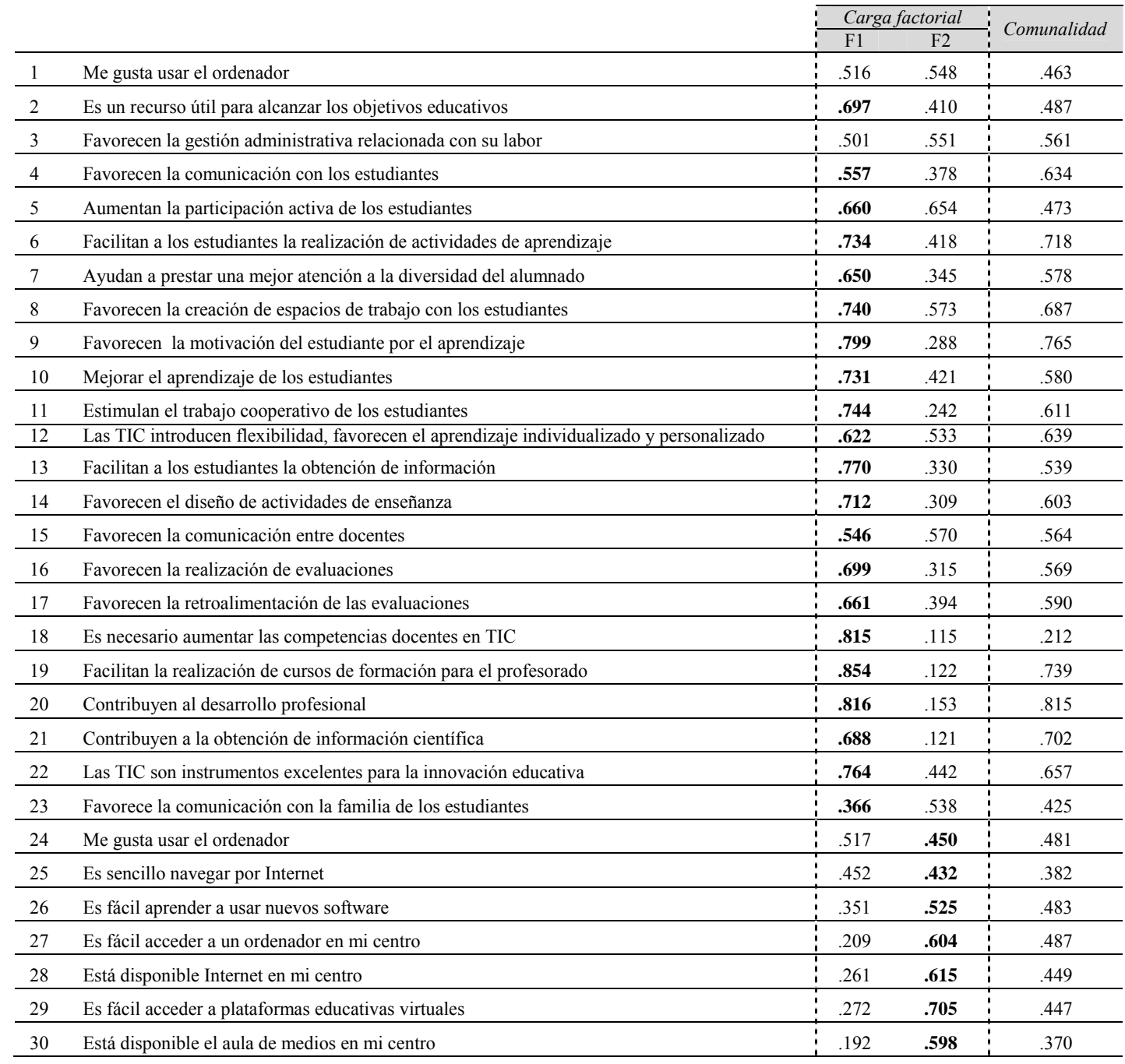

Tabla 2. Análisis factorial del cuestionario. 


\begin{tabular}{lll}
\hline Factor & Definición & Indicadores \\
\hline $\begin{array}{l}\text { Apoyo en el } \\
\text { desarrollo }\end{array}$ & Valoración respecto a la ayuda que ofrecen las & $1,2,3,4,5,6,7,8,9,10,11,12,13,14,1$ \\
$\begin{array}{l}\text { Profesional } \\
\text { Docente }\end{array}$ & procesos de enseñanza-aprendizaje & \\
\hline $\begin{array}{l}\text { Facilidad y } \\
\text { accesibilidad }\end{array}$ & Valoración con respecto al acceso y manejo de & $24,25,26,27,28,29$ y 30 \\
\hline
\end{tabular}

Tabla 3. Especificaciones del cuestionario

Para establecer la confiabilidad se obtuvo el Alfa de Cronbach por factor y global del instrumento. Este indicador demostró una adecuada confiabilidad, en todos los casos: apoyo en el desarrollo profesional y docente (.82); facilidad y disponibilidad (.84) y Global (.88).

Para obtener la información, en un primer momento se les solicitó autorización a los directores y coordinadores de secciones de los respectivos centros de Educación Permanente de Adultos, después de explicarles los objetivos del estudio y solicitarles la colaboración voluntaria. Una vez obtenida una contestación positiva, se les indicaba el número de cuestionarios que necesitarían. Junto a los cuestionarios se les enviaba un sobre con la dirección a la que debían ser devueltos dichos instrumentos de recogida de información. Para analizar los datos, se utilizaron el SPSS 17 y programas estadísticos descriptivos e inferenciales.

Con respecto a las entrevistas, decir que utilizamos un guión para homogeneizar, en la medida de lo posible, la recogida de información. Dicho guión fue validado por el mismo grupo de profesores que participó en la validación del cuestionario. Se realizaron 18 entrevistas en profundidad a coordinadores y directores de centros de educación permanente de adultos que participaron de forma voluntaria. Todas las entrevistas se grabaron en audio y tuvieron una duración media de 40 minutos, en función de la complejidad de cada una de las secuencias. Para el desarrollo de nuestro sistema categorial elaboramos un listado de categorías a priori concebidas a partir de la elaboración del cuestionario. Realizamos una primera lectura y aproximación al análisis de los datos, para comprobar si las áreas de significado que definían se observaban en todos los casos. A raíz de estas lecturas, de este análisis exploratoria de los datos, aparecieron nuevos códigos y se suprimieron otros.

\section{Resultados}

\section{El cuestionario}

Para establecer el sentido de las actitudes hacia las TIC por parte de los docentes, en un primer momento, se compararon las puntuaciones de cada factor y global contra una media teórica de $3(\mu=3)$. Las puntuaciones significativamente superiores a los de la media teórica se consideraron indicadores de una actitud positiva y las puntuaciones que fueron significativamente menores, presentaban una actitud negativa. Los datos 
obtenidos indican que en el nivel global y en los factores estudiados, los docentes manifestaban una actitud positiva hacia las TIC: Apoyo en el desarrollo profesional y docente (X: 4, 24); facilidad y accesibilidad (X: 3,75) y global (X: 3,99).

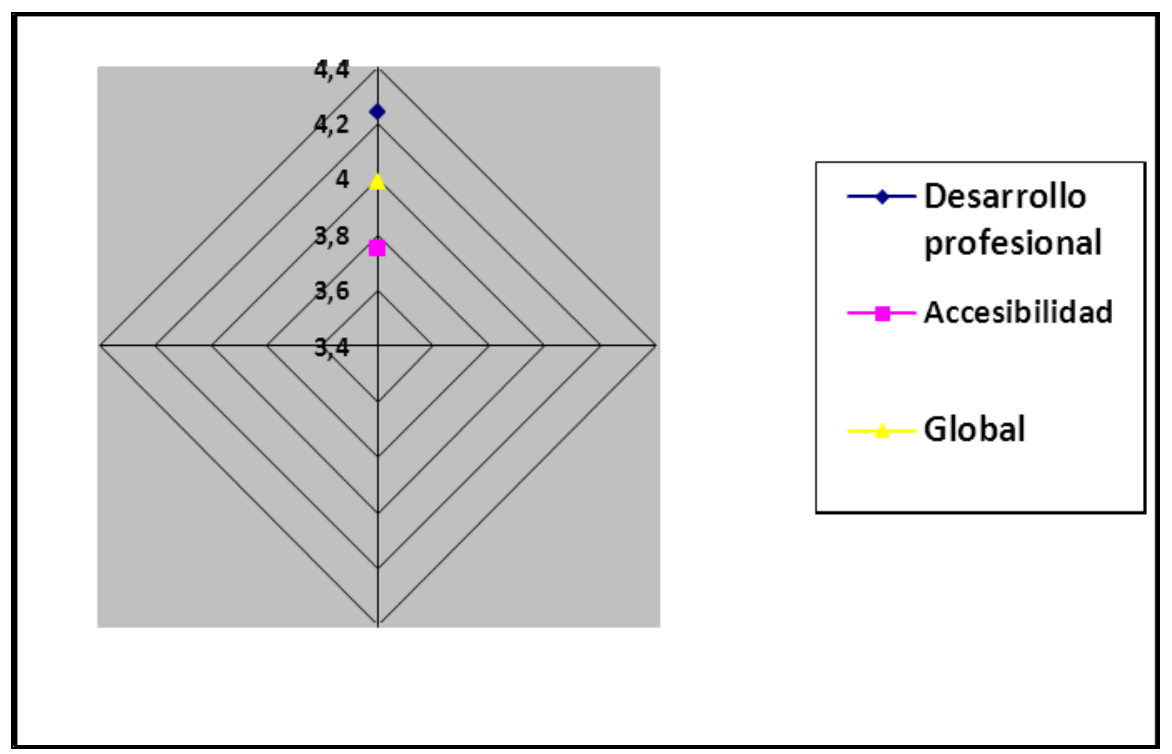

Grafico 1. Puntuaciones medias de cada factor y global

Para establecer si había diferencias significativas en las puntuaciones de los diferentes factores, se utilizó una prueba $t$ de Student para muestras relacionadas. Los resultados confirman que hay diferencias significativas en las puntuaciones de los factores, siendo mayores los correspondientes al factor Apoyo en el desarrollo profesional y docente, lo que implica que las actitudes de los docentes son más positivas en este aspecto.

\begin{tabular}{lcccc}
\hline Factores & $\boldsymbol{x}$ & $\boldsymbol{t}$ & $\boldsymbol{g l}$ & $\boldsymbol{p}$ \\
\hline Desarrollo profesional docente & 4.24 & 1.434 & 171 & .115 \\
\hline Facilidad y accesibilidad & 3.75 & 1.434 & 171 & .115 \\
\hline$p \leq .05$ & & & & \\
\hline
\end{tabular}

Tabla 4. Comparaciones de las puntuaciones de los factores 
Con respecto a las actitudes del profesorado hacia las TIC y su relación con variables como el «Género», se procedió a la realización de una prueba $t$ de Student para muestras independientes con el objeto de establecer si existían diferencias estadísticamente significativas entre las puntuaciones por factor y globales del instrumento de acuerdo con estas variables. Los resultados evidencian que no existen diferencias significativas en las actitudes hacia las TIC de acuerdo al género (Tabla 5).

\begin{tabular}{lccccc}
\hline Factores & Género & $\mathbf{x}$ & $\mathbf{t}$ & $\mathbf{g l}$ & $\mathbf{p}$ \\
\hline Apoyo en el desarrollo profesional docente & $\mathrm{F}$ & 4.22 & -.341 & 170 & .734 \\
\cline { 2 - 6 } & $\mathrm{M}$ & 4.34 & -.331 & 170 & .742 \\
\hline Facilidad y disponibilidad & $\mathrm{F}$ & 3.51 & -.638 & 170 & .526 \\
\cline { 2 - 6 } & $\mathrm{M}$ & 3.61 & -.662 & 170 & .521 \\
\hline$* \mathrm{p} \leq .05$ & & & & & \\
\hline
\end{tabular}

Tabla 5. Comparaciones por género

En lo que respecta a la variable «Edad», se establecieron dos grupos de profesores, el primero de ellos comprendía a los docentes entre 20 y 40 años, y el segundo, a los que tenían entre 41-65 años. Al igual que con la variable de género se utilizó una prueba $t$ de Student para muestras independientes, no encontrándose diferencias significativas entre los mismos, lo que permite afirmar que la edad no es una variable que establezca diferencia en las actitudes de los docentes hacia las TIC (Tabla 6).

\begin{tabular}{lcrrrr}
\hline Factores & Edad & $\mathbf{x}$ & $\mathbf{t}$ & $\mathbf{g l}$ & $\mathbf{p}$ \\
\hline Apoyo en el desarrollo profesional docente & $20-40$ & 4.42 & -.425 & 170 & .634 \\
\cline { 2 - 6 } & $41-65$ & 4.38 & & 170 & \\
\hline Facilidad y disponibilidad & $20-40$ & 4.41 & -2.84 & 170 & .004 \\
\cline { 2 - 6 } & $41-65$ & 3.91 & & 170 & \\
\hline$* \mathrm{p} \leq .05$ & & & & & \\
\hline
\end{tabular}

Tabla 6. Comparaciones por edad

Para determinar la existencia de diferencias significativas entre las actitudes hacia las TIC entre los docentes que poseen ordenador e internet en su domicilio familiar y los que no, a pesar de que los primeros son el 98\% de los encuestados, se utilizó una prueba $t$ de Student para muestras independientes donde se evidencia que las actitudes de los docentes que poseen internet en casa son significativamente mayores que los que no las poseen.

Según el curso en que imparten clases, se dividió a los docentes en dos grupos. Los que imparten los programas de Formación Básica I y II, y aquellos que ejercen la docencia en el programa de Acceso a Titulación. Se utilizó una prueba Anova (análisis de varianza) de una vía con el propósito de detectar diferencias significativas entre las 
puntuaciones de los dos grupos. Los resultados mostraron que no existieron diferencias significativas entre ellos, lo que implica que las actitudes de ambos grupos son semejantes en este aspecto (Tabla 7).

\begin{tabular}{llll}
\hline Factores & F & gl & p \\
\hline Apoyo en el desarrollo profesional docente & .260 & 2 & $.03^{1}$ \\
\hline Facilidad y disponibilidad & .442 & 2 & .004 \\
\hline Global & .731 & 2 & .025 \\
\hline $\mathrm{p} \leq .05$ & & & \\
\hline
\end{tabular}

Tabla 7. Comparaciones en las puntuaciones de acuerdo con el grado impartido

Al mismo tiempo, los datos reflejan que los profesores se encuentran motivados, ya que reconocen que aunque las TIC suponen un esfuerzo adicional a su carga de trabajo, es un hecho que estimula y favorece el aprendizaje a los estudiantes (Tabla 8).

\begin{tabular}{lcc}
\hline & $\begin{array}{c}\text { Totalmente } \\
\text { De Acuerdo }\end{array}$ & $\begin{array}{c}\text { De } \\
\text { Acuerdo }\end{array}$ \\
\hline Favorecen la comunicación con los estudiantes & $46 \%$ & $36 \%$ \\
\hline Aumentan la participación activa de los estudiantes & $51 \%$ & $29 \%$ \\
\hline Facilitan a los estudiantes la realización de actividades de aprendizaje & $41 \%$ & $48 \%$ \\
\hline Ayudan a prestar una mejor atención a la diversidad del alumnado & $37 \%$ & $49 \%$ \\
\hline Favorecen la creación de espacios de trabajo con los estudiantes & $29 \%$ & $52 \%$ \\
\hline Favorecen la motivación del estudiante por el aprendizaje & $41 \%$ & $45 \%$ \\
\hline Mejorar el aprendizaje de los estudiantes & $35 \%$ & $39 \%$ \\
\hline
\end{tabular}

Tabla 8. Aspectos favorecedores del aprendizaje de los estudiantes

Un aspecto importante a tener en cuenta es la necesidad de formación en lo referente a las TIC, a través de cursos de formación, a pesar de que ello suponga un trabajo adicional a su carga docente. En este sentido, el $91 \%$ del profesorado encuestado, manifiesta la importancia de aumentar las competencias docentes en TIC.

\section{Entrevistas}

El análisis de los discursos confirma y amplia los resultados obtenidos en otras investigaciones (Canales \& Marqués, 2007; Fernández Batanero \& Bermejo, 2012). En este sentido, se extrajeron una serie de factores de buenas prácticas educativas con apoyo de las TIC, y que fueron agrupados en 2 grandes apartados coincidiendo con las dimensiones del cuestionario: 


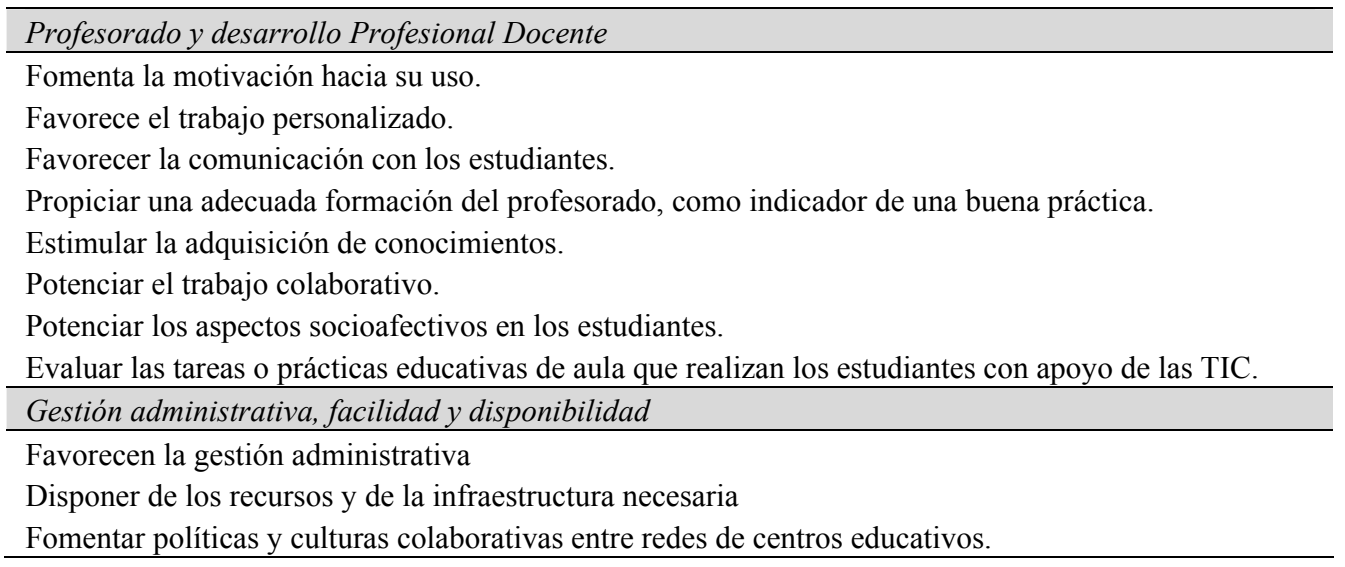

Cuadro 1. Factores que favorecen buenas prácticas educativas

Todos los directores y coordinadores de secciones entrevistados coinciden que sus respectivos centros reúnen condiciones tecnológicas óptimas para desarrollar procesos de aprendizaje con apoyo de TIC, así como que estas poseen un gran potencial para favorecer la gestión administrativa.

«En la actualidad la dotación tecnológica de que disponemos es muy buena, aunque como todos sabemos la tecnología cambia día a día, y ya mismo, algunos de nuestros ordenadores se quedaran anticuados» (Coord. 4).

«Antes el papeleo era impresionante. Ahora gracias a las tecnologías nuestro trabajo es más llevadero y al mismo tiempo más eficaz. En mi caso que llevo cerca de 26 años en adultos, he podido ver toda esta transformación» (Coord. 6).

La técnica didáctica más usada en comunicación con TIC en sus centros, según los entrevistados, es la llamada «uno-a-uno», donde la comunicación se establece básicamente entre dos personas. Es por tanto individual y personalizada. Le sigue la técnica «uno-solo», que se basa en el paradigma de la recuperación de la información de Internet.

«No debemos olvidar que son personas adultas y que muchas de ellas tienen una baja cualificación, de ahí que la estrategia que más utilicemos en nuestro centro con relación a la comunicación con TIC sea muy personalizada» (Coord. 9).

Del análisis de contenido se desprende que entre los factores más destacados se encuentran: «favorecer la comunicación con los estudiantes» y el «estimular el aprendizaje o la adquisición del conocimiento» usando las TIC en las prácticas de aula, donde todos los coordinadores entrevistados consideran que el uso de las TIC incrementa la calidad de los aprendizajes, situación que los motiva a impulsar su uso en las distintas actividades, utilizando la red Internet para la formación de los estudiantes, vinculando los contenidos y los objetivos curriculares con las actividades de enseñanza y aprendizaje apoyadas en las TIC, y trabajando los libros de texto complementando su uso con software educativo y/o recursos electrónicos. 
«Creo que el papel principal que tienen la TIC es su capacidad de motivación y papel tan importante que ejercen para fomentar la comunicación entre este tipo de alumnado indistintamente que cumplan muchas funciones más» (Coord. 2).

Un factor a tener en cuenta es la formación del profesorado. Se concluye que hay falta de conocimientos en los profesores, ya sea de carácter técnico para algunos y pedagógicos para otros, quizás por problemas generacionales o problemas con la propia formación, pero son temáticas que pueden impedir realizar buenas prácticas educativas con apoyo de las TIC. Este aspecto, se ve cumplimentado, en la medida de lo posible, con otro factor que sale a la luz y es la presencia en casi todos los centros educativos de la existencia de algún docente que actúa de ariete en la alfabetización digital del profesorado y por extensión de toda la comunidad educativas. Prácticamente todos los entrevistados coinciden en el perfil innovador y comprometido del profesorado de educación de adultos. A ello hay que añadirle, que el ser equipos de docentes pequeños facilita la coordinación en el diseño, aplicación y evaluación de tareas y actividades.

«El profesorado de adultos es un profesor diferente. Somos equipos muy pequeños y nos conocemos todos como si fuésemos hermanos. Somos maestros especiales. Somos maestros muy comprometidos y con un carácter puramente compensador. Somos maestros que nos implicamos mucho y somos conscientes de la importancia de las TIC. De ahí, que al ser centros pequeños seamos una gran familia, donde uno se siente apoyado por los otros» (Coord. 13).

Un factor importante a destacar es la potenciación en los estudiantes de tareas con TIC, orientadas al trabajo autónomo y cooperativo, a la capacidad de búsqueda de la información y al fortalecimiento de las habilidades de investigador usando las tecnologías. Se matiza que se estimula mucho la reflexión y la discusión entre los estudiantes.

Los profesores manifiestan que a pesar de que sus respectivos centros se encuentran insertos en sus correspondientes contextos sociales, las TIC no suelen facilitar la comunicación con los agentes sociales y posibles familiares de esta tipología de alumnado.

«Disponemos de páginas web en la sección, pero no es muy concurrida. Para la comunicación tenemos que emplear otras técnicas como es el boca a boca o plasmar la información en los establecimientos y lugares de ocio del pueblo» (Coord. 7).

\section{Conclusiones y discusión}

Los resultados del estudio permitieron llegar a las siguientes conclusiones:

- Existencia, de forma general, de una panorámica bastante positiva en relación con las actitudes del profesorado andaluz de educación permanente de adultos hacia las TIC.

- Los resultados evidencian que no existen diferencias significativas en las actitudes hacia las TIC de acuerdo al género y la edad. Hallazgos coinciden 
con estudios anteriores como los realizados por Valdés et al. (2012), a pesar de que la reciente encuesta PIAAC (2013), estable una diferencia de 5 puntos entre hombre y mujeres.

- Las actitudes de los docentes estudiados hacia las TIC no se ve influenciada por factores tales como el nivel educativo de impartición (planes educativos de formación básica I y II).

- El hecho de contar con recursos informáticos suficientes, actualizados y con un funcionamiento correcto, es un factor esencial y un requisito necesario para optar a la posibilidad de aplicar las tecnologías en los contextos educativos (Baraja, Scheuermann, \& And Kikis, 2002; Van Braak, 2002).

- Las actitudes hacia las TIC son positivas en los dos factores estudiados (Apoyo en el desarrollo profesional docente; y facilidad y disponibilidad). Esto puede asociarse a la existencia de profesorado cualificado que actúa de motor de arranque a la hora de trabajar con TIC. Ello, constituye un aspecto muy importante, pues el asesoramiento directo influye en última instancia en las decisiones de los docentes de usar o no las TIC en sus prácticas educativas. Al mismo tiempo, el asesoramiento contribuye al desarrollo de una coordinación y cooperación entre docentes facilitando la labor de aplicación de las TIC, y aportando diferentes puntos de vista y un enriquecimiento derivado de la colaboración entre profesionales.

- Entre los factores más importantes para proporcionar buenas prácticas educativas con apoyo de TIC se encuentran el «Fomenta la motivación hacia su uso», «Favorece el trabajo personalizado», «favorecer la comunicación con los estudiantes» $\mathrm{y}$ «estimular el aprendizaje o la adquisición del conocimiento».

- La formación del profesorado y unas condiciones tecnológicas óptimas para iniciar procesos de innovación e implementación de las TIC aparecen como factores importantes para desarrollar buenas prácticas educativas, aspecto coincidente con estudios como los realizados por (Valverde, Garrido \& Fernández Sánchez, 2010)

- La mayoría de los profesores declaran que las TIC contribuyen al desarrollo profesional y facilitan la realización de cursos de formación para el profesorado, coincidiendo estos resultados con los datos aportados por Boza, Tirado \& Guzmán, (2010), sobre la inserción de las TIC en los centros educativos andaluces y las creencias del profesorado sobre la integración de las TIC y la formación que han recibido sobre las mismas.

Para finalizar decir que coincidimos con Valdés et al. (2012, 4), cuando afirma «que la consistencia de los estudios que refieren la presencia de actitudes positivas hacia las TIC en los docentes parecen indicar que las dificultades en el uso de los mismos, no se puede explicar con base en las actitudes; lo cual abre la necesidad de explorar otros factores como las competencias en el uso de las mismas y sus concepciones pedagógicas». Ahora bien, somos conscientes que la opinión de los 
docentes sobre el potencial didáctico de las TIC va a condicionar el uso que hagan de estas Herramientas en su práctica docente.

\section{Limitaciones del estudio}

Como ya hemos señalado en trabajos anteriores, debemos decir que el carácter exploratorio de este estudio aconseja una interpretación prudente de los resultados, fundamentalmente a la hora de generalizar sus resultados a otros contextos. Pero el carácter inicial abre nuevos interrogantes de especial interés para la investigación en este campo (extender el estudio a todo el profesorado, diseñar y construir nuevos instrumentos que permitan un análisis más pormenorizado de las diferentes actitudes y competencias profesionales de los docentes, incorporar nuevos contextos...).

\section{Recomendaciones}

Desarrollar acciones para favorecer la frecuencia de uso de las TIC por parte de los docentes de educación permanente de Adultos, como una estrategia que beneficie la presencia de actitudes más positivas de los mismos hacia éstas.

\section{Referencias bibliográficas}

ÁREA, M. (2008). La innovación pedagógica con TIC y el desarrollo de las competencias informacionales y digitales. Investigación en la Escuela, 64, 5-17. Recuperado de http://dialnet.unirioja.es/servlet/articulo?codigo=2593487 $(03 / 05 / 2011)$

ÁREA, M. (2010). El proceso de integración y uso pedagógico de las TIC en los centros educativos. Un estudio de casos. Revista Educación, 352, 77-97. Recuperado de http://www.revistaeducacion.mec.es/re352/re352_04.pdf $(08 / 02 / 2012)$

BARAJAS, M., SCHEUERMANN, F. \& KIKIS, K. (2002). Critical indicators of innovative practices in ICT-supported learning. Paper presented at the «Improving learning through technology: Opportunities for all» PROMETEUS Conference (Paris 2002). Recuperado de http://www.prometeus.org/PromDocs/hb_arttic_be_08-10-02_11-36-03.doc $(23 / 12 / 2010)$

BLASCO, J. et al. (2010). "Validación mediante el método Delphi de un cuestionario para conocer las experiencias e interés hacia las actividades acuáticas con especial atención al Winsurf. Agora para la educación física y el deporte. 12, 75-94.

BOZA, A., TIRADO, R. \& GUZMÁN M. (2010). Creencias del profesorado sobre el significado de la tecnología en la enseñanza: influencia para su inserción en los centros docentes andaluces. Revista Relieve, 16(1), 1- 24. 
CABERO, J. (1991). Actitudes de los profesores hacia los ordenadores y la informática. In M. Cebrian (Dir.). Medios y recursos didácticos. (pp. 85-98). Málaga: Secretariado de Publicaciones de la Universidad de Málaga.

CANALES, R. \& MARQUÉS, P. (2007). Factores de buenas prácticas educativas con apoyo de las TIC Análisis de su presencia en tres centros educativos. Educar, 39, 115-133.

CASTAÑO, C. (1994). Análisis y evaluación de las actitudes de los profesores hacia los medios de enseñanza. Bilbao: Universidad del País Vasco.

CHICKERING A. W. Y GAMSON Z. (1987). Seven Principles for Good Practise in Undergraduate Education. American Association for Higher Education Bulletin (march): Washington, DC.

COLL, C., MAURI, T. \& ONRUBIA, J. (2008). Análisis de los usos reales de las TIC en contextos educativos formales: una aproximación socio-cultural. Revista Electrónica de Investigación Educativa, 10 (1), 1-18. Recuperado de http://redie.uabc.mx/voll no1/contenido-coll2.html (13/11/2010).

EPPER, R. M. \& BATES, A. W. (2004). Enseñar al profesorado como utilizar la tecnología. Buenas prácticas de instituciones líderes. Barcelona: UOC.

FERNÁNDEZ BATANERO, J. M. Y BERMEJO, B. (2012). Actitudes docentes hacia las TIC en centros de buenas prácticas educativas con orientación inclusiva. Enseñanza \& Teaching. Revista interuniversitaria de didáctica, 30 (1), 45-61.

GALANOULI, D.; MURPHY, C. \& GARDNER, J. (2004). Teachers' perception of the effectiveness of ICT-competence training. Computers and Education, 43, 63-79.

GARGallo, B., SUÁrEZ, J., BELlOCH, C. \& AL. (2004). Perfiles actitudinales de los profesores ante las TIC e incidencia de las actitudes sobre su uso. Conferencia presentada en Virtual Educación (Barcelona, 2004). Recuperado de http://e-spacio.uned.es/fez/view.php?pid=bibliuned:19584 (13/01/2013).

HINOJO, F. C. \& FERNÁNDEZ GRANADA, F. (2002). Diseño de escalas de actitudes para la formación del profesorado en tecnología. Comunicar, 19, 120-125.

MOOIJ, T. (2004). Optimising ICT effectiveness in instruction and learning: multilevel transformation theory and a pilot project in secondary education. Computers and Education, 42, 25-44.

ORELLANA, N., ALMERICH, G., BELLOCH C. Y DÍAZ, I. (2009). La actitud del profesorado ante las TIC: Un aspecto clave para la integración. Ponencia presentada en el X Congreso Nacional de Investigación Educativa. Veracruz: COMIE. Recuperado de http://www.uv.es/ bellochc/doc\%20UTE/VE2004_5_6.pdf $(29 / 12 / 2012)$

PALOMO, R., RUIZ, J Y SÁNCHEZ, J. (2006). Las TIC como agente de innovación educativa. Consejería de Educación de la Junta de Andalucía. Recuperado de http://www.juntadeandalucia.es/averroes/publicaciones/nntt/TIC_como_agentes_in novacion.pdf. 
RODRÍGUEZ MONDEJAR (2000). Las actitudes del profesorado hacia la informática. Pixel-Bit. Revista de Medios y Educación,15, 35-49.

SÁEZ LÓPEZ, J. M. (2010). Actitudes de los docentes respecto a las TIC a partir del desarrollo de una práctica reflexiva. Escuela Abierta, 13, 37-54.

TEJEDOR, F. J., GARCÍA-VALCÁRCEL A. \& PRADA, S. (2009). Medida de actitudes del profesorado universitario hacia la integración de las TIC. Comunicar, 33, 115-124.

VALDÉS, A., ANGUlO, J., NIEBlAS, E. H., ZAMBRANO, L. \& ARREOLA, C. (2012). Actitudes de docentes de secundaria hacia el uso de las TIC. Investigación educativa duranguense, 12, 4-10.

VALVERDE, J., GARRIDO, Ma C. \& FERNÁNDEZ SÁNCHEZ, R. (2010). Enseñar y aprender con tecnologías: un modelo teórico para las buenas prácticas con TIC. Teoría de la Educación, 11 (3), 203-229.

VAN BRAAK J. (2001). Factors influencing the use of computer mediated communication by teachers secondary schools. Computers and Education, 36, 4157.

\section{Correspondencia con los autores}

José María FERNÁNDEZ BATANERO

Facultad de Ciencias de la Educación

$\mathrm{C} /$ Pirotecnia s/n

41013Sevilla (España)

email: batanero@us.es

José Antonio TORRES GONZÁLEZ

Facultad de Humanidades y Ciencias de la Educación

Campus las Lagunillas s/n

23071 Jaén (España)

email: jtorres@ujaen.es 